for example, where $H=\left(\mathrm{d} F_{1}-\mathrm{d} F_{2}\right) / \mathrm{d} G$, with the expected value $E\left(p_{1}\right)=\Pi_{1}$, and variance

$$
\sigma^{2}\left(p_{1}\right)=\frac{\int H^{2} \mathrm{~d} F-\left(\int H \mathrm{~d} F\right)^{2}}{n\left[\int\left(\mathrm{d} F_{1}-\mathrm{d} F_{2}\right) H\right]^{2}}
$$

A considerable choice of $H$ is possible. Thus, considering for definiteness the density case, we define the unweighted least-squares estimator of $\Pi_{1}$ by the choice $\mathrm{d} G=d x$, $H=f_{1}-f_{2}$. We should, howover, expect weighted estimators to be better. In fact, because the variance of $\mathrm{d} F_{s}{ }_{s}$ is $f d x / n$, we should try to choose $\mathrm{d} G=f d x$; the term $\int H \mathrm{~d} F$ in the expression for $\sigma^{2}\left(p_{1}\right)$, arising from the covariance of $\mathrm{d} F_{s}(x)$ and $\mathrm{d} F_{s}(y)$, then vanishes, and $\sigma^{2}\left(p_{1}\right)$ becomes identical with the reciprocal of the information function $I\left(\Pi_{1}\right)$. If we take $\mathrm{d} G=\Pi_{0} \mathrm{~d} F_{1}+\left(1-\Pi_{0}\right) \mathrm{d} F_{2}$, then this weighting will be most efficient when $\Pi_{0}$ is close to $\Pi_{1}$. Thus if we suspect $\Pi_{1}$ to be near $1, \frac{1}{2}$ or 0 , suitable choices for $\Pi_{0}$ would be $1, \frac{1}{2}$ or 0 , respectively. An alternative to $\frac{1}{2}\left(\mathrm{~d} F_{1}+\mathrm{d} F_{2}\right)$ is the geometric mean of $\mathrm{d} F_{1}$ and $\mathrm{d} F_{2}$, and another is $\max \left(\mathrm{d} F_{1}, \mathrm{~d} F_{2}\right)$. The geometric mean has some convenience over the arithmetic mean in theoretical investigations of efficiency (for example, for $f_{1}$ and $f_{2}$ normal), but the latter (or alternatively $\left.\max \left(\mathrm{d} F_{1}, \mathrm{~d} F_{2}\right)\right)$ has the advantage of approximating to the maximum likelihood estimator, for any value of $\Pi_{1}$, in both the extreme cases of $f_{1}$ and $f_{2}$ well separated and of $f_{1} \rightarrow f_{2}$. It has been shown by Hill ${ }^{1}$ that the information function $I\left(\Pi_{1}\right)$ for $\Pi_{1}$ may be written $n\left[1-S\left(\Pi_{1}\right)\right] /\left(\Pi_{1} \Pi_{2}\right)$, where

$$
S\left(\mathrm{II}_{1}\right)=\int\left(f_{1} f_{2} / \mathrm{f}\right) \mathrm{d} x
$$

If $f_{1}$ and $f_{\mathrm{a}}$ differ by some parameter $\mu$ (for example, the mean), and $\Delta \mu_{1}=\mu_{2}-\mu_{1}$, then also as $f_{1} \rightarrow f_{2}$,

$$
I\left(\Pi_{1}\right) \sim n(\Delta \mu)^{2} I(\mu)
$$

where $I(\mu)$ is the information function for $\mu$. Thus for the maximum likelihood estimator $\hat{\Pi}_{1}$ the variance, which is $\Pi_{1} \Pi_{2} / n$ for $f_{1}$ and $f_{2}$ well separated, is $1 /\left[n(\Delta \mu)^{2} I(\mu)\right]$ as $f_{1} \rightarrow f_{2}$.

In the case of further unknown parameters $\theta_{j}$ the problem is obviously more complex, because of the non-linear dependence of $f$ on $\theta_{j}$; but linearization of the estimation equations may sometimes be convenient. For example, for two populations with unknown parameters $\theta_{1}, \theta_{2}$, respectively, we might adopt the standard leastsquares procedure of taking provisional values $v_{1}, v_{2}$, say, so that in the "sum of squares" to be minimized we write in the numerator

$$
f \sim f_{2}\left(v_{2}\right)+\mathrm{II}_{1}\left(f_{1}\left(v_{1}\right)-f_{2}\left(v_{2}\right)\right)+\varphi_{1} \partial f_{1} / \partial v_{1}+\varphi_{2} \partial f_{2} / \partial v_{2}
$$

in the unknowns $\Pi_{1}, \varphi_{1}$ and $\varphi_{2}$, where

$$
\varphi_{1}=\Pi_{1}\left(\theta_{1}-v_{1}\right), \varphi_{2}=\Pi_{2}\left(\theta_{2}-v_{2}\right)
$$

(retaining the provisional values $v_{1}$ and $v_{2}$ in the weightingfunction $\mathrm{d} G$ ).

$$
\begin{aligned}
& \text { M. S. Bartlett } \\
& \text { P. D. M. Macdonalo }
\end{aligned}
$$

Departmont of Biomathernaties,

University of Oxford.

Received December 13, 1967.

' Hill, B. M., ,J. Amer. Stat. Assoc., 58, 918 (1963).

\section{Speculations on the Use of Orthonormal Functions in the Study of Morphogenesis}

THE recent proposal ${ }^{1}$ to analyse shapes of biological objects by means of orthonormal functions opens up some interesting possibilitics. In the work cited, a particular set of two-dimensional orthonormal functions synthesized from Walsh" functions which take only the values +1 and -1 was used. As is well known, however, there is a vast number of other complete sets of orthonormal functions and, in fact, they could be constructed indefinitely. There are also standard linear methods for transforming from one set of orthonormal functions to another; these procedures can be realized as computer programmes.

The Walsh-derived functions are most suitable as an initial set because of their simplicity. Their characteristic chequerboard patterns, however, are not the most natural to use for the analysis of familiar kinds of biological objects; this was indicated by the fact ${ }^{1}$ that, for good accuracy, as many as 256 terms might be nooded in the expansion for a leaf. It might be possible to construct a different orthonormal set of basic patterns which would be more "lcaf-like" in shape; ideally, a development series of leaves would all be adequately deseribed by merely two or three terms, say. What is required then is to devise computer programmes which, for a givon series of input shapes, would find the best orthonormal set-using the transformation procedures referred to--best in the sense that fewest terms would be required for adequate description. When this had boen achieved, one might hope to discover laws of a general type from the experimental data, for example, by studying the changos of the weights (that is, coefficients in the orthonormal expansion) that occur in development or because of experimentally applied external agents. If this were achieved, the basic patterns would presumably have genuine biological significance.

What follows is rather more speculative. It is well known that most of the standard orthonormal functions of mathematical physics (sinusoidal or Bessel, for example) arise naturally in the solution of differential equations with boundary constraints. Thus a given set of orthonormal functions characterizes a given differential equation with its associated boundary conditions. If then an orthonormal set which was particularly adequato in the sense of the previous paragraph was found, this set might well have a special significance and be indicative of some differential equation or, maybe, difference equation or other type of equation, which characterizes in a general way the development of the patterns concerned. It might thus be possible, merely from examining the optimum orthonormal sets generated by the computer programmes, to draw conclusions about or even to derive the fundamental laws-in the form of differential equations or something similar-which govern the development of form.

Another issue, which it may be fruitful to investigate, is the biological meaning, if any, of the maximal weights previously discussed ${ }^{1}$. If one attempted to reconstruct a form from the maximal weights directly, one would fail, because in general the constructed function would have other values than 0 and 1 . Nevertheless, this function, because it contains all the information about the degrees of resemblance of the form concerned to the basic pattern forms, should have a significance which might be usable--especially in those cases where the number of basic patterns effectively used is very small, as discussed previously.

It would also be useful to generalize the orthonormal expansion mothod by allowing the form function to have more than two values. For example, a representation of the veins of a leaf might be included with a value of 2 being assigned at locations on a vein. This would provide a three-valued function, but many-valued funetions could be used also for other purposes such as giving the distribution of colour as well as shape of an object, when a suitable numerical coding of the colours had been chosen.

Metamathematics Unit.

Bernard Meltzer

University of Edinburgh.

Receivcd December 5, 1987.

' Meltzer, B., Searle, N. H., and Brown, R., Nature, 216, 32 (1967).

${ }^{2}$ Walsh, T. I., Amer. J. Math., 45, 5 (1923). 